\title{
A domain-theoretic approach to Brownian motion and general continuous stochastic processes
}

\author{
Paul Bilokon Abbas Edalat \\ Department of Computing \\ Imperial College London \\ \{paul.bilokon,a.edalat\}@imperial.ac.uk
}

\begin{abstract}
We introduce a domain-theoretic framework for continuous-time, continuous-state stochastic processes. The laws of stochastic processes are embedded into the space of maximal elements of the normalised probabilistic power domain on the space of continuous interval-valued functions endowed with the relative Scott topology. We use the resulting $\omega$-continuous bounded complete dcpo to define partial stochastic processes and characterise their computability. For a given continuous stochastic process, we show how its domain-theoretic, i.e., finitary, approximations can be constructed, whose least upper bound is the law of the stochastic process. As a main result, we apply our methodology to Brownian motion. We construct a partial Wiener measure and show that the Wiener measure is computable within the domain-theoretic framework.
\end{abstract}

Categories and Subject Descriptors F.1.1 [Theory of Computation]: Models of Computation-Computability theory; F.4.1 [Mathematical Logic and Formal Languages]: Mathematical Logic-Computability theory

\section{General Terms Algorithms, Design, Theory}

Keywords Brownian motion; Wiener measure; Domain theory; Stochastic processes; Computability

\section{Introduction}

This work is motivated by a desire to improve our understanding of stochastic processes, particularly in the light of recursion theory. In recent decades, major advances in stochastic calculus have been motivated by applications in the rapidly expanding field of quantitative finance. Stochastic processes have many other important applications, notably in filtering problems, stochastic approaches to deterministic boundary value problems, optimal stopping, and stochastic control [30].

Permission to make digital or hard copies of all or part of this work for personal or classroom use is granted without fee provided that copies are not made or distributed for profit or commercial advantage and that copies bear this notice and the full citation on the first page. Copyrights for components of this work owned by others than ACM must be honored. Abstracting with credit is permitted. To copy otherwise, or republish, to post on servers or to redistribute to lists, requires prior specific permission and/or a fee. Request permissions from permissions@acm.org.

CSL-LICS 2014, July 14-18, 2014, Vienna, Austria.

Copyright (c) 2014 ACM 978-1-4503-2886-9...\$15.00.

http://dx.doi.org/10.1145/2603088.2603102
Several schools of computable analysis have addressed the subject of measure theory and integration. The computability of measures and integration on the unit interval was studied in the light of Type2 Theory of Effectivity (TTE) [35]. The computability of measures and set-theoretical operations was examined in the setting of a computable measure space [38, 39]. Computable probability frameworks were used to study Martin-Löf and dynamical randomness [18]. In a separate strand of literature, interval-valued and fuzzyvalued random variables have been considered, and there have also been extensions of stochastic integration to interval-valued and setvalued processes $[27,40]$.

This article follows the tradition of applying domain theory [33] to classical analysis, which started with applications to dynamical systems, measures and fractals [7] and integration [5]. In this approach the classical spaces are realised as a subset of maximal elements of an $\omega$-continuous dcpo, where the set of maximal elements is endowed with the relative Scott topology. By embedding the set of probability measures of any locally compact second countable metric space into the set of maximal elements of the probabilistic power domain [19] of the upper space of the metric space [7] a new theory of approximation of measures was obtained. This resulted in a generalisation of the Riemann integral to the so-called R-integral [5]. When the embedding is onto the set of maximal elements of such a dcpo, then the classical space is precisely a complete metrisable separable metric space [24].

More generally, as in the context of the present paper, when a separable metric space is homeomorphic to a $G_{\delta}$ subset of the maximal elements of an $\omega$-continuous dcpo endowed with the Scott topology, the space of probability measures of the metric space endowed with the weak topology is homeomorphic to a subset of the maximal elements of the probabilistic power domain of the $\omega$ continuous dcpo [9]. This result establishes a connection between the classical measure theory and domain theory with applications in various areas [8].

In [6], domain theory has been applied to discrete time stochastic processes. Here we follow a different path and develop a more general approach. We consider continuous time, continuous space stochastic processes through the prism of domain theory. Not only does this theoretical apparatus allow us to examine the question of computability, it naturally yields new approaches to computation of stochastic processes by constructing a new data type for representing them.

The plan for this paper is as follows. In Sec. 2 we present some domain-theoretic and topological preliminaries. In Sec. 3, we introduce a domain-theoretic framework for continuous-time, continuous-state stochastic processes. This is realised by considering them with the underlying compact-open topology of the space 
of trajectories and embedding them into the set of maximal elements of the normalised probabilistic power domain of the space of Scott continuous interval-valued functions or trajectories, which extend the classical notion of trajectories of stochastic processes in the domain-theoretic setting. We derive a necessary and sufficient condition for the least upper bound of an increasing sequence of simple valuations in the normalised probabilistic power domain to be supported on the maximal elements, i.e., be in effect the law of a stochastic process.

Given a Borel measure supported on the maximal elements of a bounded complete $\omega$-continuous dcpo, we construct an increasing sequence of simple valuations on the normalised probabilistic power domain of the dcpo whose least upper bound is the given measure. In particular, for a given continuous stochastic process, this yields a domain-theoretic approximation by partial stochastic processes (Sec. 4). We then formulate a notion of computability for partial stochastic processes which is used to define domaintheoretic computability for a classical stochastic process (Sec. 5).

As one of our main results, we apply our methodology to Brownian motion and its law, the Wiener measure (Sec. 6). Brownian motion is the stochastic process $W$ defined by the following three properties: (i) $W_{0}=0$, (ii) the function $t \rightarrow W_{t}$ is almost surely everywhere continuous, (iii) $W$ has independent increments with $W_{t}-W_{s}$ normally distributed with expected value 0 and variance $t-s$. The Wiener measure of a basic point-open set of continuous functions from $[0,1]$ to $\mathbb{R}$, i.e. a set of the form

$$
\left\{f \mid a_{i}<f\left(t_{i}\right)<b_{i}, 0=t_{0}<t_{1}<\ldots,<t_{n}=1\right\},
$$

is given by

$$
\frac{1}{\sqrt{\pi^{n} \prod_{i=1}^{n}\left(t_{i}-t_{i-1}\right)}} \int_{a_{1}}^{b_{1}} \ldots \int_{a_{n}}^{b_{n}} e^{\sum_{j=1}^{n} \frac{\left(x_{j}-x_{j-1}\right)^{2}}{t_{j}-t_{j-1}}} d x_{n} \ldots d x_{1},
$$

where $x_{0}:=0$. The computability of the Wiener measure and Brownian motion has previously been studied by Fouché $[4,13]$. In particular, when all $a_{i}, b_{i}$ and $t_{i}$ are computable real numbers, the real number given by (1) is also computable.

The Lebesgue-type integral of a functional with respect to the Wiener measure is known as the Wiener integral whose computation presents significant challenges even for the simplest functionals [31]; research has focussed on the computation of several special cases $[3,21]$. Wiener measure and integration play a major rôle in stochastic analysis due to their association with Brownian motion and have found major applications in quantum physics [34] such as Feynman integration.

Due to the central role played by the Wiener measure in stochastic analysis and theoretical physics, the question of its computability attracted the attention of researchers working in the field of computable analysis. This question was addressed by Fouché. In [13], he considered ways in which the Brownian motion can be approximated by oscillations which are encoded by finite binary strings of high descriptive complexity. This enabled him to deduce recursive properties of this stochastic process. In [14], he showed that Brownian motion can be computed from an infinite binary string which is complex in the sense of Kolmogorov-Chaitin. He presents a direct construction of complex oscillations from algorithmically real numbers based on ideas underlying the construction of Gaussian processes as spirals in Gaussian Hilbert spaces. This is similar to Wiener's Fourier analytic approach in that the Brownian motion is regarded as a random signal with random, normally distributed amplitudes. An investigation of the computability of this construction is presented in [4].

In this paper we develop a domain-theoretic method for approximating stochastic processes. We then show that the Wiener measure is domain-theoretically computable by using, among other things, a result by Paul Lévy. This provides an alternative proof of the computability of the compact-open sets to that discovered by Fouché [13] and enables us to create a domain-theoretic approximation for Brownian motion.

The application of the domain-theoretic machinery to this problem creates many possibilities for further work, some of which are listed in Sec. 7.

\section{Preliminaries}

We assume that the reader is already familiar with foundations of general topology and domain theory $[1,16]$. In this paper we strive to use the mainstream notation and terminology. We shall use the term 'base' for topological bases and 'basis' for domain-theoretic bases. The interior of a set $A$ is denoted by $A^{\circ}$ and its closure by $\bar{A}$. By domain we mean an $\omega$-continuous dcpo. We shall be primarily concerned with bounded complete domains (sometimes called continuous Scott domains).

The interval domain of the real line is the collection of compact intervals $\mathbf{I}:=\{[a, b] \mid a, b \in \mathbb{R} \wedge a \leq b\}$ ordered by reverse subset inclusion. It is a bounded complete domain. For directed $A \subseteq \mathbf{I}$, $\sqcup A=\bigcap A ; I \ll J \Leftrightarrow J \subseteq I^{\circ}$; and $\{[p, q] \mid p, q \in \mathbb{Q} \wedge p \leq q\}$ is a countable basis for $\mathbb{I} \mathbb{R}$. Similarly, we can replace $\mathbb{R}$ with a compact interval $C \subseteq \mathbb{R}$ to obtain the interval domain of $C, \mathbf{I} C$.

Let $X$ be a locally compact Hausdorff space, $O(X)$ be its lattice of open subsets, and $L$ be a bounded complete domain. For $O \in O(X)$ and $a \in L$, a single-step function is the continuous map

$$
a \chi_{o}(x)= \begin{cases}a, & \text { if } x \in O \\ \perp, & \text { otherwise }\end{cases}
$$

where $\perp$ is the bottom element. A step function is a join of a bounded finite collection of single-step functions. The set $[X \rightarrow L]$ of all continuous functions $g: X \rightarrow L$ is a bounded complete domain with respect to the pointwise order induced by $L$ with a basis consisting of step functions. We shall refer to the step functions made up of basic open sets of $X$ and basis elements of $L$ as basic step functions. When regarding $[X \rightarrow L]$ as a topological space, we imply that it is endowed with the Scott topology. We shall require the following results from [12]:

Proposition 2.1. For all $f \in[X \rightarrow L]$ :

1. For every $O \in O(X)$ and every $a \in L, O \ll f^{-1}(\uparrow a)$ iff a $\chi_{O} \ll f$;

2. For every finite family $O_{i} \in O(X)$ and $a_{i} \in L$ such that $O_{i} \ll$ $f^{-1}\left(\uparrow a_{i}\right)$ for $i=1, \ldots, n$, we have that $\bigsqcup_{i=1}^{n} a_{i} \chi_{o_{i}} \ll f$;

3. $f=\bigsqcup\left\{a \chi_{o} \mid O \ll f^{-1}(\uparrow a)\right\}$.

A valuation on the topological space $X$ is a function $v: O(X) \rightarrow$ $[0, \infty)$ with the properties (i) $v(\emptyset)=0$; (ii) $v(O)+v(U)=v(O \cup$ $U)+v(O \cap U)$; (iii) $O \subseteq U \Rightarrow v(O) \leq v(U)$ for $O, U \in O(X)$. A continuous valuation is a valuation such that whenever $A \subseteq O(X)$ is a directed set (wrt $\subseteq$ ) of open sets of $X$, then $v\left(\cup_{O \in A} O\right)=$ $\sup _{O \in A} v(O)$. For any $b \in X$, the point valuation $\delta_{b}: O(X) \rightarrow[0, \infty)$ defined by

$$
\delta_{b}(O)= \begin{cases}1, & \text { if } b \in O ; \\ 0, & \text { otherwise. }\end{cases}
$$

Any finite linear combination $\sum_{i=1}^{n} r_{i} \delta_{b_{i}}$ with $r_{i} \in[0, \infty), 1 \leq i \leq n$, is a continuous valuation on $X$. It is called a simple valuation. The (normalised) probabilistic power domain $\mathbf{P} X\left(\mathbf{P}^{1} X\right)$ of $X$ consists 
of the set of continuous valuations $v$ on $X$ (with $v(X)=1$ ) and is ordered pointwise: $v \sqsubseteq v^{\prime}$ iff, for all $O \in O(X), v(O) \leq v^{\prime}(O)$. If $X$ is an $\omega$-continuous depo with $\perp$, then $\mathbf{P}^{1} X$ is also an $\omega$-continuous dcpo with bottom element $\delta_{\perp}$ and has a basis consisting of simple valuations.

Let $X$ and $Y$ be two topological spaces. By $C(X, Y)$ we shall denote the set of all continuous functions from $X$ to $Y$. If $K$ is a compact subset of $X$ and $U$ is an open subset of $Y$, define the compact-open subset

$$
V(K, U):=\{f \in C(X, Y) \mid f(K) \subseteq U\} .
$$

These sets form a subbase for a topology on $C(X, Y)$ called the compact-open topology. We denote the corresponding basic open sets by

$$
V\left(K_{1}, \ldots, K_{n} ; U_{1}, \ldots, U_{n}\right)=\bigcap_{i=1}^{n} V\left(K_{i}, U_{i}\right), \quad n \in \mathbb{N}^{+} .
$$

When referring to $C(X, Y)$ as a topological space, we shall always be implying the compact-open topology.

It is well known that if $X$ is a locally compact Hausdorff space then, for any base $\mathcal{B}$ of $X$, the family $\mathcal{B}_{c}:=\{U \in \mathcal{B} \mid \bar{U}$ is compact $\}$ is also a base for $X$. We can therefore require that a base for such a space contain only relatively compact sets (i.e. those whose closures are compact). It is possible to relate topological bases for $X$ and $Y$ to one for $C(X, Y)$.

Lemma 2.2. Let $X$ be a locally compact Hausdorff space with a base $\mathcal{B}$ consisting of relatively compact sets. Let $Y$ be a topological space with a base $\mathcal{U}$. Then a base for $C(X, Y)$ is given by the sets

$$
V\left(\overline{O_{1}}, \ldots, \overline{O_{n}} ; U_{1}, \ldots, U_{n}\right), \quad n=1,2, \ldots,
$$

with $O_{1}, \ldots, O_{n} \in \mathcal{B}$ and $U_{1}, \ldots, U_{n} \in \mathcal{U}$.

As a consequence, if both $X$ and $Y$ are second countable, then $C(X, Y)$ is also second countable. We can similarly relate a topological base for $X$ and a domain-theoretic basis for $L$ to a domaintheoretic basis for $[X \rightarrow L]$.

Lemma 2.3. Let $X$ be a locally compact Hausdorff space with a base $\mathcal{B}$ consisting of relatively compact sets. Let $L$ be a bounded complete domain with a basis $C$. Then a basis for $[X \rightarrow L]$ is given by step functions of the form

$$
\bigsqcup_{i=1}^{n} a_{i} \chi_{o_{i}}, \quad n=1,2, \ldots,
$$

with $O_{1}, \ldots, O_{n} \in \mathcal{B}$ and $a_{1}, \ldots, a_{n} \in C$.

\section{The domain-theoretic model}

Let $(\Omega, \mathcal{F}, \mathbb{P})$ be a probability space. Let $T:=[0,1]$ and $S:=\mathbb{R}$, endowed with their usual topologies, represent, respectively, the time space and state space. Let $\mathcal{G}$ be the Borel $\sigma$-algebra of $S$. We consider the stochastic process $X: T \times \Omega \rightarrow S$. The map $X_{t}: \Omega \rightarrow S: \omega \mapsto X(t, \omega)$ is a $(\mathcal{F}, \mathcal{G})$-measurable function for $t \in T$.

For a topological space $Z$ let $\mathbf{M} Z$ be the collection of Borel measures on $Z$. Let $C(T, S)$ denote the space of all continuous functions from $T$ into $S$, endowed with the compact-open topology. We are given the law of a stochastic process, $\mu \in \mathbf{M} C(T, S)$. This is a measure on the measurable space $(C(T, S), \mathcal{B})$, where $\mathcal{B}$ is the Borel $\sigma$-algebra corresponding to the compact-open topology. We shall construct a domain-theoretic approximation for $\mu$.
Consider the map $s: C(T, S) \rightarrow[T \rightarrow \mathbf{I} S]$ defined by $s:$ $f \mapsto \lambda x .\{f(x)\}$. The topologies on the domain and range are, respectively, the compact-open topology and Scott topology.

Each function $f: T \rightarrow \mathbf{I} S$ corresponds to a pair of functions $f^{-}, f^{+}: T \rightarrow S$ with $f(x)=:\left[f^{-}(x), f^{+}(x)\right]$. Recall that $f \in$ $[T \rightarrow \mathbf{I} S]$ iff $f^{-}$and $f^{+}$are, respectively, lower and upper semicontinuous.

The image $M:=s(C(T, S))$ is a proper subset of the set $\operatorname{Max}[T \rightarrow \mathbf{I} S$ ] of maximal elements of $[T \rightarrow \mathbf{I} S]$. It is well known, for example, that the function

$$
g(x):= \begin{cases}\{-1\}, & x<1 / 2 \\ {[-1,1],} & x=1 / 2 \\ \{1\}, & x>1 / 2\end{cases}
$$

is a maximal element of $[T \rightarrow \mathbf{I} S$ ], while it is not an image of any classical function in $C(T, S)$ under $s$.

Lemma 3.1. 1. The map s is continuous.

2. For any positive integer $n, i=1, \ldots, n$, let $O_{i}$ be open and $a_{i} \in$ IS such that $a_{i}$ is not maximal, i.e., $a_{i}^{-}<a_{i}^{+}$in $\left[a_{i}^{-}, a_{i}^{+}\right]:=a_{i}$. Then

$$
s\left(\bigcap_{i=1}^{n} V\left(\overline{O_{i}}, a_{i}^{\circ}\right)\right)=\bigcap_{i=1}^{n} \uparrow a_{i} \chi_{O_{i}} \cap M .
$$

Proof. 1. Take any open $O \subseteq T$ and $a \in \mathbf{I} S$. Then

$$
\begin{gathered}
s^{-1}\left(\uparrow a \chi_{o}\right) \stackrel{\text { Prop. } 2.1}{=} \\
s^{-1}\left(\left\{g \in[T \rightarrow \mathbf{I} S] \mid O \ll g^{-1}(\uparrow a)\right\}\right)= \\
s^{-1}\left(\left\{g \in[T \rightarrow \mathbf{I} S] \mid \bar{O} \subseteq g^{-1}\left(\left\{b \in \mathbf{I} S \mid b \subseteq a^{\circ}\right\}\right)\right\}\right)= \\
V\left(\bar{O}, a^{\circ}\right) .
\end{gathered}
$$

2.

$$
\begin{gathered}
\bigcap_{i=1}^{n} \uparrow a_{i} \chi \chi_{i} \cap M \stackrel{\text { Prop. } 2.1}{=} \\
\bigcap_{i=1}^{n}\left\{g \in[T \rightarrow \mathbf{I} S] \mid O_{i} \ll g^{-1}\left(\uparrow a_{i}\right)\right\} \cap M= \\
\bigcap_{i=1}^{n}\left\{g \in[T \rightarrow \mathbf{I} S] \mid \overline{O_{i}} \subseteq g^{-1}\left(\left\{b \in \mathbf{I} S \mid b \subseteq a_{i}^{\circ}\right\}\right)\right\} \cap M= \\
s\left(\bigcap_{i=1}^{n}\left\{f \in C(T, S) \mid f\left(\overline{O_{i}}\right) \subseteq a_{i}^{\circ}\right\}\right)=s\left(\bigcap_{i=1}^{n} V\left(\overline{O_{i}}, a_{i}^{\circ}\right)\right) .
\end{gathered}
$$

Proposition 3.2. The map s is a topological embedding.

Proof. We have already ascertained (Lemma 3.1 (1)) that $s$ is continuous. By Lemma 2.2, a base for $C(T, S)$ is given by sets of the form $\bigcap_{i=1}^{n} V\left(\overline{O_{i}}, K_{i}^{\circ}\right), n$ being a positive integer, $O_{i}$ open intervals, and $K_{i}$ compact intervals. By Lemmas 3.1 (2) and 2.3, the image of such a basic open set is a basic open set of the relative Scott topology. Since images and arbitrary unions commute, the image of any open set under $s$ is an open set of the relative Scott topology.

In summary, the relative Scott topology on the proper subset $M$ of the maximal elements of $[T \rightarrow \mathbf{I} S]$ coincides with the compactopen topology. This gives rise to the subspace of the embedded classical functions. 
For all $I=[c, d] \in \mathbf{I} S$, let $|I|:=d-c$ be the diameter of $I$. Similarly, for all $f \in[T \rightarrow \mathbf{I} S]$, let $|f|:=\max _{t \in T}|f(t)|$. Call this the diameter of $f$. For all $n \in \mathbb{N}^{+}$, define

$$
U_{n}:=\bigcup\{\uparrow g \mid g \in[T \rightarrow \mathbf{I} S] \text { a step function, }|g|=1 / n\} .
$$

Proposition 3.3. $C(T, S)$ is homeomorphic to a $G_{\delta}$ subset (i.e. a countable intersection of open sets) of $[T \rightarrow \mathbf{I} S]$.

Proof. This is an adaptation of [7, Prop. 5.9] to our setting. $M=$ $\cap_{n=1}^{\infty} U_{n}$, which is a countable intersection of open sets.

As $T$ is compact Hausdorff and second countable, the function space $C(T, S)$ is separable. Whenever a separable metric space is homeomorphic to a $G_{\delta}$ subset of a domain endowed with its Scott topology, the space of probability measures of the metric space endowed with the weak topology is homeomorphic with a subset of the maximal elements of the probabilistic power domain of that domain [9].

Thus let $\mathbf{M}^{1} C(T, S)$ be the space of probability measures (normalised measures) on $C(T, S)$ with the weak topology, i.e. the coarsest topology on the set of normalised measures such that the functional

$$
F_{g}: \mathbf{M}^{1} C(T, S) \rightarrow \mathbb{R}, \quad \mu \mapsto \int g d \mu
$$

is continuous for all bounded continuous maps

$$
g: C(T, S) \rightarrow \mathbb{R}
$$

Let

$$
e: \mathbf{M}^{1} C(T, S) \rightarrow \mathbf{P}[T \rightarrow \mathbf{I} S]
$$

be defined by

$$
e(\mu)=\mu \circ s^{-1} .
$$

By the results of [9, Sec. 3], $e$ embeds $\mathbf{M}^{1} C(T, S)$ into the set of maximal elements of $\mathbf{P}^{1}[T \rightarrow \mathbf{I} S]$.

The image of $e$ consists of continuous valuations on $[T \rightarrow \mathbf{I} S]$ whose unique extension to a measure is supported on $M$. This provides a domain-theoretic framework for classical stochastic processes. In fact, $\mathbf{P}^{1}[T \rightarrow \mathbf{I} S]$ is $\omega$-continuous with a basis of simple valuations. It follows that for any $\mu \in \mathbf{M}^{1} C(T, S)$ there is an increasing chain of simple valuations $\left(v_{n}\right)_{n \in \mathbb{N}}$ in $\mathbf{P}^{1}[T \rightarrow \mathbf{I} S]$ with

$$
e(\mu)=\bigsqcup_{n \in \mathbb{N}} v_{n} \text {. }
$$

The simple valuations provide finite approximations to $\mu$, the law of the stochastic process.

Let $v \in \mathbf{P}[T \rightarrow \mathbf{I} S]$ be a continuous valuation. Then $v$ extends uniquely to a probability measure $v^{*}$ on $[T \rightarrow \mathbf{I} S$ ] [2]. We say that $v$ is supported on $M$ if $v^{*}(M)=1$. We shall now derive a necessary and sufficient condition for the least upper bound of an increasing sequence of simple valuations to be supported on the maximal elements of $[T \rightarrow \mathbf{I} S$ ], i.e. being in effect the law of a stochastic process.

Proposition 3.4. The continuous valuation $v$ is supported on $M$ iff for all $n \in \mathbb{N}^{+}, v\left(U_{n}\right)=1\left(U_{n}\right.$ as defined in Proposition 3.3.)

Proof. By the properties of measures,

$$
v^{*}(M)=v\left(\bigcap_{n=1}^{\infty} U_{n}\right)=\lim _{n \rightarrow \infty} v\left(U_{n}\right) .
$$

If, for each $n \in \mathbb{N}^{+}, v\left(U_{n}\right)=1$, then $\lim _{n \rightarrow \infty} v\left(U_{n}\right)=1$. Therefore $v$ is supported on $M$. On the other hand, if $v$ is supported on $M$, then $\lim _{n \rightarrow \infty} v\left(U_{n}\right)=1$. Since $\left\{U_{n}\right\}_{n \in \mathbb{N}^{+}}$is a decreasing sequence of sets, for all $n \in \mathbb{N}^{+}, v\left(U_{n}\right)=1$.

A valuation on the probabilistic power domain of a domain is maximal iff it is supported in the set of maximal elements (Proposition 5.18 in [7] and Theorem 8.6 in [23]). Therefore, if $v$ is supported on $M$, the valuation $v$ is maximal.

For a simple valuation $v:=\sum_{j=1}^{n} r_{j} \delta_{g_{j}}, n \in \mathbb{N}^{+}$, and $l \in \mathbb{R}^{+}$define the $l$-mass of $v$ by $m_{l}(v):=\sum_{j=1}^{n}\left\{r_{j}|| g_{j} \mid<l\right\}$.

Proposition 3.5. Suppose that $v_{1} \sqsubseteq v_{2} \sqsubseteq v_{3} \sqsubseteq \ldots$ is an increasing chain of simple valuations in $\mathbf{P}^{1}[T \rightarrow \mathbf{I} S]$ with

$$
v_{i}:=\sum_{j=1}^{n_{i}} r_{i j} \delta_{g_{i j}},
$$

$n_{i} \in \mathbb{N}^{+}$. Define $v:=\bigsqcup_{n \in \mathbb{N}^{+}} v_{n}$. Then $v$ is supported on the subspace of the embedded classical functions iff, for all $n \in \mathbb{N}^{+}$, there exists $N \in \mathbb{N}^{+}$such that

$$
m_{1 / n}\left(v_{N}\right)>1-1 / n
$$

Proof. Suppose that, for all $n \in \mathbb{N}^{+}$, the required $N$ exists. Fix any $n \in \mathbb{N}^{+}$and $\epsilon>0$. Take some $k \in \mathbb{N}$ such that

$$
1 / k<\min \{\epsilon, 1 / n\} \text {. }
$$

Then there is some $N \in \mathbb{N}^{+}$such that

$$
1-m_{1 / k}\left(v_{N}\right)<1 / k<\epsilon .
$$

By construction, functions of diameter less than $1 / n$ are in $U_{n}$, so we have $1-v_{N}\left(U_{n}\right)<\epsilon$. Therefore $v\left(U_{n}\right)=\sup _{i} v_{i}\left(U_{n}\right)=1$, and, by Proposition 3.4, $v$ is supported on $M$.

Now suppose that $v$ is supported on $M$. Fix any $n \in \mathbb{N}^{+}$. By Proposition 3.4, $v\left(U_{n}\right)=1$. Therefore there exists $N$ such that

$$
1-v_{N}\left(U_{n}\right)<1 / n,
$$

and so

$$
m_{1 / n}\left(v_{N}\right)>1-1 / n
$$

\section{The approximation of a stochastic process}

We now know the existence of an approximating domain-theoretic sequence for the law $\mu$ of a stochastic process, but we haven't yet demonstrated how to construct it explicitly. The rest of this section is dedicated to such a construction, which is a main result of the paper. While we could adopt the method in Sec. 3 of [9], we will present a general result that holds for any bounded complete domain.

Let $D$ be a bounded complete domain with a countable basis

$$
B:=\left(b_{1}, b_{2}, \ldots\right)
$$

closed under finite bounded suprema. The topological space $X$ is embedded into the set of maximal elements of $D$, the embedding being $s$, thus $M \subseteq \operatorname{Max}(D)$.

We will recursively define a sequence of finite lists, which is used to construct an increasing chain of simple valuations converging to $\mu$. Define the finite lists

$$
A_{0}:=\left[a_{1}^{0}:=\perp\right]
$$


and, for $n \in \mathbb{N}$,

$$
A_{n+1}=\left[b_{n+1} \sqcup a_{l_{1}}^{n}, \ldots, b_{n+1} \sqcup a_{l_{L_{n}}}^{n}, a_{1}^{n}, \ldots, a_{K_{n}}^{n}\right],
$$

where

$$
A_{n}=\left[a_{1}^{n}, \ldots, a_{K_{n}}^{n}\right]
$$

and $\left[a_{l_{1}}^{n}, \ldots, a_{l_{L_{n}}}^{n}\right]$ is the sublist of $A_{n}$ consisting of those elements that have an upper bound with $b_{n+1}$. $\left(L_{n} \leq K_{n}\right.$.)

For example,

$$
\begin{gathered}
A_{1}=\left[b_{1}, \perp\right] ; \\
A_{2}= \begin{cases}{\left[b_{2} \sqcup b_{1}, b_{2}, b_{1}, \perp\right]} & \text { if } b_{2} \sqcup b_{1} \text { exists, } \\
{\left[b_{2}, b_{1}, \perp\right]} & \text { otherwise. }\end{cases}
\end{gathered}
$$

Further, for $n \in \mathbb{N}$,

$$
v_{n}:=\sum_{i=1}^{K_{n}} r_{i}^{n} \delta_{a_{i}^{n}},
$$

where

$$
r_{i}^{n}:=e(\mu)\left(\left[\uparrow a_{i}^{n} \backslash \bigcup_{k=1}^{i-1} \uparrow a_{k}^{n}\right]\right) .
$$

We begin by proving that $\left\{v_{n}\right\}$ is increasing. Recall that if $L$ is a dcpo, then for any $a, b \in L$ such that $a \sqcup b$ exists,

$$
\uparrow(a \sqcup b)=(\uparrow a) \cap(\uparrow b) .
$$

If $L$ is bounded complete, then for any $a, b \in L$ such that $a \sqcup b$ does not exist,

$$
(\uparrow a) \cap(\uparrow b)=\emptyset .
$$

Proposition 4.1. The sequence of simple valuations $\left(v_{n}\right)_{n \in \mathbb{N}}$ is an increasing chain, i.e., for all $n \in \mathbb{N}, v_{n} \sqsubseteq v_{n+1}$.

Proof. We employ the modification [5] of the splitting lemma [19] for the normalised probabilistic power domain: we need to show the existence of the nonnegative numbers (called transport numbers) $t_{i, j}^{n}$ for

such that

$$
i=1, \ldots, K_{n}, j=1, \ldots, K_{n+1},
$$

$$
\begin{aligned}
& \text { for a fixed } i: \sum_{j=1}^{K_{n+1}} t_{i, j}^{n}=r_{i}^{n}, \\
& \text { for a fixed } j: \sum_{i=1}^{K_{n}} t_{i, j}^{n}=r_{j}^{n+1},
\end{aligned}
$$

and $t_{i, j}^{n} \neq 0$ implies $a_{i}^{n} \sqsubseteq a_{j}^{n+1}$.

We claim that these requirements are satisfied by defining the transport numbers as follows. If $b_{n+1} \sqcup a_{i}^{n}$ exists, then $i=l_{j_{i}}$ for a unique $j_{i} \in\left\{1, \ldots, L_{n}\right\}$, and we define

$$
\begin{aligned}
t_{i, j_{i}}^{n} & :=r_{j_{i}}^{n+1}, \\
t_{i, L_{n}+i}^{n} & :=r_{L_{n}+i}^{n+1}, \\
t_{i, j}^{n} & :=0
\end{aligned}
$$

for all $j \notin\left\{j_{i}, L_{n}+i\right\}$. If $b_{n+1} \sqcup a_{i}^{n}$ does not exist, then we define

$$
\begin{aligned}
t_{i, L_{n}+i}^{n} & :=r_{L_{n}+i}^{n+1}, \\
t_{i, j}^{n} & :=0
\end{aligned}
$$

for all $j \neq L_{n}+i$ (Figure 1).
The transport numbers thus defined are nonnegative by virtue of being measures of sets. If $i=l_{j_{i}}$ for some $j_{i} \in\left\{1, \ldots, L_{n}\right\}$, then

$$
\begin{aligned}
& \sum_{j=1}^{K_{n+1}} t_{i, j}^{n}=t_{i, j_{i}}^{n}+t_{i, L_{n}+i}^{n}=r_{j_{i}}^{n+1}+r_{L_{n}+i}^{n+1} \\
& =e(\mu)\left(\left[\uparrow a_{j_{i}}^{n+1} \backslash \bigcup_{k=1}^{j_{i}-1} \uparrow a_{k}^{n+1}\right]\right) \\
& +e(\mu)\left(\left[\uparrow a_{L_{n}+i}^{n+1} \backslash \bigcup_{k=1}^{L_{n}+i-1} \uparrow a_{k}^{n+1}\right]\right) \\
& =e(\mu)\left(\left[\uparrow\left(b_{n+1} \sqcup a_{i}^{n}\right) \backslash \bigcup_{k=1}^{j_{i}-1} \uparrow\left(b_{n+1} \sqcup a_{l_{k}}^{n}\right)\right]\right) \\
& +e(\mu)\left(\left[\uparrow a_{i}^{n} \backslash\left\{\bigcup_{k=1}^{L_{n}} \uparrow\left(b_{n+1} \sqcup a_{l_{k}}\right) \cup \bigcup_{k=1}^{i-1} \uparrow a_{k}^{n}\right\}\right]\right) \\
& =e(\mu)\left(\left[\left(\left(\uparrow b_{n+1}\right) \cap\left(\uparrow a_{i}^{n}\right)\right) \backslash \bigcup_{k=1}^{i-1} \uparrow a_{k}^{n}\right]\right) \\
& +e(\mu)\left(\left[\left(\left(\uparrow b_{n+1}\right)^{c} \cap\left(\uparrow a_{i}^{n}\right)\right) \backslash \bigcup_{k=1}^{i-1} \uparrow a_{k}^{n}\right]\right) \\
& \stackrel{\text { finite }}{=} e(\mu)\left(\left\{\left[\left(\uparrow b_{n+1}\right) \cap\left(\uparrow a_{i}^{n}\right)\right) \backslash \bigcup_{k=1}^{i-1} \uparrow a_{k}^{n}\right]\right\} \\
& \left.\cup\left\{\left[\left(\left(\uparrow b_{n+1}\right)^{\mathrm{c}} \cap\left(\uparrow a_{i}^{n}\right)\right) \backslash \bigcup_{k=1}^{i-1} \uparrow a_{k}^{n}\right]\right\}\right) \\
& =e(\mu)\left(\left[\uparrow a_{i}^{n} \backslash \bigcup_{k=1}^{i-1} \uparrow a_{k}^{n}\right]\right)=r_{i}^{n} .
\end{aligned}
$$

If $i \notin\left\{l_{1}, \ldots, l_{L_{n}}\right\}$, then $\left(\uparrow b_{n+1}\right) \cap\left(\uparrow a_{i}^{n}\right)=\emptyset$ and

$$
\begin{aligned}
\sum_{j=1}^{K_{n+1}} t_{i, j}^{n} & =t_{i, L_{n}+i}^{n}=r_{L_{n}+i}^{n+1} \\
& =e(\mu)\left(\left[\uparrow a_{L_{n}+i}^{n+1} \backslash \bigcup_{k=1}^{L_{n}+i-1} \uparrow a_{k}^{n+1}\right]\right) \\
& =e(\mu)\left(\left[\uparrow a_{i}^{n} \backslash\left\{\bigcup_{k=1}^{L_{n}} \uparrow\left(b_{n+1} \sqcup a_{l_{k}}^{n}\right) \cup \bigcup_{k=1}^{i-1} \uparrow a_{k}^{n}\right\}\right]\right) \\
& =e(\mu)\left(\left[\uparrow a_{i}^{n} \backslash\left\{\bigcup_{k=1}^{L_{n}}\left(\left(\uparrow b_{n+1}\right) \cap\left(\uparrow a_{l_{k}}^{n}\right)\right) \cup \bigcup_{k=1}^{i-1} \uparrow a_{k}^{n}\right\}\right]\right) \\
& =e(\mu)\left(\left[\uparrow a_{i}^{n} \backslash \bigcup_{k=1}^{i-1} \uparrow a_{k}^{n}\right]\right)=r_{i}^{n} .
\end{aligned}
$$

The remaining relationships required for the splitting lemma to apply hold trivially.

Note that in our case the bounded complete domain $C(T, S)$, with $T=[0,1], S=\mathbb{R}$, has a countable basis closed under finite suprema. It is given by the step functions obtained from rationalvalued intervals. Let us consider convergence. First, some auxiliary results.

Proposition 4.2. [9, Lemma 3.1] Let $v_{1}$ and $v_{2}$ be continuous valuations on a topological space $X$. Suppose $B \subseteq O(X)$ is a base which is closed under finite intersections. If $v_{1}(O)=v_{2}(O)$ for all $O \in B$, then $v_{1}=v_{2}$. 


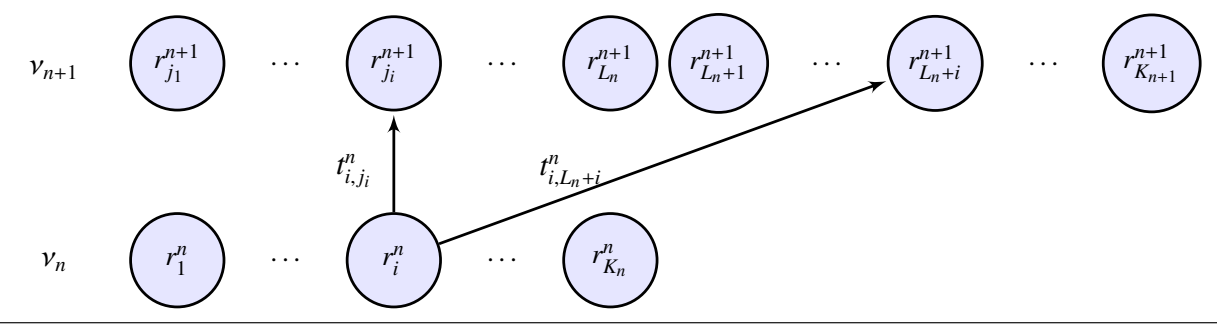

Figure 1. Transport numbers

Recall that the way-below relation in a bounded complete domain $D$ is called meet-stable if, for all $x, y, z \in D$,

$$
(x \ll y \wedge x \ll z) \Rightarrow x \ll y \sqcap z .
$$

For example, $\mathbf{I} S$ is meet-stable.

Proposition 4.3. [11, Corollary 5.12] If $X$ is a topological space with a meet-stable continuous lattice of open sets and L is a bounded complete domain with a meet-stable way-below relation, then for any step function $f \in[X \rightarrow L]$ we have:

$$
\uparrow f=\bigcup\{\uparrow h \mid f \ll h, h \text { is a step function }\} .
$$

By the interpolation property of $\ll$ we obtain, for any step function $f \in[X \rightarrow L]:$

\section{Corollary 4.4.}

$$
\uparrow f=\bigcup\{\uparrow h \mid f \ll h, h \text { is a basic step function }\} .
$$

Theorem 4.5. The supremum of the approximating chain $\left(v_{n}\right)_{n \in \mathbb{N}}$ of simple valuations gives the approximated measure: $\bigsqcup_{n \in \mathbb{N}} v_{n}=e(\mu)$.

Proof. The countable basis $B$ for our domain $D$ gives rise to the topological base for its Scott topology, consisting of the sets $\uparrow b_{k}$ for each $b_{k} \in B, k \in \mathbb{N}^{+}$. Since $B$ is closed under finite suprema, the topological base is closed under finite intersections.

By Prop. 4.2 it suffices to ascertain that $\bigsqcup_{n \in \mathbb{N}} v_{n}\left(\uparrow b_{k}\right)=e(\mu)\left(\uparrow b_{k}\right)$ for each $b_{k} \in B$.

For each $n \in \mathbb{N}$,

$$
\begin{aligned}
& v_{n}\left(\uparrow b_{k}\right)=\sum_{i=1}^{K_{n}} e(\mu)\left(\left[\uparrow a_{i}^{n} \backslash \bigcup_{l=1}^{i-1}\left(\uparrow a_{l}^{n}\right)\right]\right) \delta_{a_{i}^{n}}\left(\uparrow b_{k}\right) \\
& =\sum_{i: b_{k} \ll a_{i}^{n}} e(\mu)\left(\left[\uparrow a_{i}^{n} \backslash \bigcup_{l=1}^{i-1}\left(\uparrow a_{l}^{n}\right)\right]\right) \\
& \underset{\text { additivity }}{\operatorname{countable}} e(\mu)\left(\bigcup_{i: b_{k} \ll a_{i}^{n}}\left[\uparrow a_{i}^{n} \backslash \bigcup_{l=1}^{i-1}\left(\uparrow a_{l}^{n}\right)\right]\right) \\
& \leq e(\mu)\left(\uparrow b_{k}\right)
\end{aligned}
$$

by monotonicity of measures, since

$$
\bigcup_{i: b_{k} \ll a_{i}^{n}}\left[\uparrow a_{i}^{n} \backslash \bigcup_{l=1}^{i-1}\left(\uparrow a_{l}^{n}\right)\right] \subseteq s^{-1}\left(\uparrow b_{k}\right) .
$$

Furthermore, we claim that $\bigsqcup_{n \in \mathbb{N}} v_{n}=e(\mu)$ since for all $\epsilon>0$ there exists $N \in \mathbb{N}$ such that

$$
e(\mu)\left(\uparrow b_{k}\right)-v_{N}\left(\uparrow b_{k}\right)<\epsilon .
$$

Indeed, by Prop. 4.4, we can find a decreasing (in way-below relation) sequence $\left(b_{m_{i}}\right) \subseteq B, i, m_{i} \in \mathbb{N}^{+}$, such that $\uparrow b_{k}=\bigcup_{i}\left(\uparrow b_{m_{i}}\right)$. By the properties of measures, there exists $N_{0} \in \mathbb{N}$ such that

$$
e(\mu)\left(\uparrow b_{k}\right)-e(\mu)\left(\uparrow b_{m_{N_{0}}}\right)<\epsilon .
$$

By construction,

$$
v_{m_{N_{0}}}\left(\uparrow b_{k}\right) \geq e(\mu)\left(\uparrow b_{m_{N_{0}}}\right)>e(\mu)\left(\uparrow b_{k}\right)-\epsilon .
$$

We take $N:=m_{N_{0}}$ and the result follows.

We can therefore think about the valuations $v_{n}$ as partial stochastic processes, which approximate and generate $\mu$ in the limit.

\section{Computable stochastic processes}

In this section we will categorise stochastic processes that are domain-theoretically computable. We fix the topological bases for $T=[0,1]$ and $S=\mathbb{R}$ consisting of either rational or dyadic open intervals. By Lemmas 2.2 and 2.3, these induce countable topological bases on $C(T, S)$ and $[T \rightarrow \mathbf{I} S]$.

Definition 5.1. An increasing chain of simple valuations $v_{0} \sqsubseteq v_{1} \sqsubseteq$ $v_{2} \sqsubseteq \ldots$, where for each $i \in \mathbb{N}, v_{i}=\sum_{i=1}^{n_{i}} r_{i j} \delta_{g_{i j}}$, is effective if for each $i, n_{i} \in \mathbb{N}$ is recursively given, $r_{i 1}, \ldots, r_{i n_{i}}$ are computable, and $g_{i 1}, \ldots, g_{i n_{i}}$ are effectively given.

Definition 5.2. A stochastic process is (domain-theoretically) computable if it is the least upper bound of an effective chain of simple valuations that satisfies the effective version of Proposition 3.5. That is, there exists a total recursive function $\phi: \mathbb{N} \rightarrow \mathbb{N}$ such that, for each $i \in \mathbb{N}$, gives $N:=\phi(i)$ as in Proposition 3.5.

Lemma 5.3. Given a measure $\mu$, let $\mathcal{A}$ be a family of $\mu$-measurable sets that is closed under finite intersections and such that the measure $\mu(A)$ of each $A \in \mathcal{A}$ is a computable real number. Then the following are also computable real numbers:

1. $\mu\left(\bigcup_{i=1}^{n} A_{i}\right)$ for each $n \in \mathbb{N}^{+}, A_{1}, \ldots, A_{n} \in \mathcal{A}$;

2. $\mu\left(A_{1} \backslash A_{2}\right)$ for $A_{1}, A_{2} \in \mathcal{A}$;

3. $\mu\left(A \backslash\left(\bigcup_{i=1}^{n} A_{i}\right)\right)$ for each $n \in \mathbb{N}^{+}, A_{1}, \ldots, A_{n} \in \mathcal{A}$.

Proof. 1. By the inclusion-exclusion principle,

$$
\begin{gathered}
\mu\left(\bigcup_{i=1}^{n} A_{i}\right)=\sum_{i=1}^{n} \mu\left(A_{i}\right)-\sum_{1 \leq i<j \leq n} \mu\left(A_{i} \cap A_{j}\right)+ \\
\sum_{1 \leq i<j<k \leq n} \mu\left(A_{i} \cap A_{j} \cap A_{k}\right)-\ldots+(-1)^{n-1} \mu\left(\bigcap_{i=1}^{n} A_{i}\right) .
\end{gathered}
$$

Each of the finite intersections appearing in this equation is in $\mathcal{A}$, so their measures are computable real numbers. The 
result follows since computable real numbers are closed under addition and subtraction.

2. By finite additivity of measures,

$$
\mu\left(A_{1} \backslash A_{2}\right)=\mu\left(A_{1} \cap\left(A_{2}^{\mathrm{c}}\right)\right)=\mu\left(A_{1}\right)-\mu\left(A_{1} \cap A_{2}\right) .
$$

$A_{1} \cap A_{2} \in \mathcal{A}$, so $\mu\left(A_{1} \cap A_{2}\right)$ is computable. The result follows since the difference of two computable real numbers is also computable.

3. A straightforward application of the proofs of parts 1 and 2 .

We assume we have an effectively given basis $B=\left(b_{1}, b_{2}, \ldots\right)$ of $[[0,1] \rightarrow \mathbb{I} \mathbb{R}]$, which we can construct by using the step functions of the function space with rational or dyadic intervals. We then use $B$ to effectively define the sequence of finite subsets $A_{n}$, with $n \in \mathbb{N}$, of $B$ recursively and define the weights $r_{i}^{n}$ on elements of $A_{n}$ and the simple valuations $v_{n}$ as in (6).

Theorem 5.4. Suppose that, for each $n \in \mathbb{N}, i=1, \ldots, K_{n}$, $e(\mu)\left(\uparrow a_{i}^{n}\right)$ is a computable number. Then $v_{0} \sqsubseteq v_{1} \sqsubseteq v_{2} \sqsubseteq \ldots$ is an effective chain and its supremum is $\mu$, in effect a domaintheoretically computable stochastic process.

Proof. By assumption, the countable basis $B$ (equation (5)) is closed under finite suprema, and as a consequence of Lemma 5.3 and our assumption that $e(\mu)\left(\uparrow a_{i}^{n}\right)$ is a computable number, the measure of each crescent set (7), $r_{i}^{n}$, is a computable number. Thus the weights in (6) are computable and Definition 5.1 is satisfied. For $\phi(i)$ in Definition 5.2 we take the first integer that satisfies the inequality (4). Proposition 4.1 and Theorem 4.5 ensure that this operation is computable. Thus Definition 5.2 is also satisfied.

For each basic open set

$$
V:=V\left(\left[u_{1}, v_{1}\right], \ldots,\left[u_{N}, v_{N}\right] ;\left(c_{1}, d_{1}\right), \ldots,\left(c_{N}, d_{N}\right)\right),
$$

with $u_{i}<v_{i}$ for $1 \leq i \leq N$, define

$$
\square V:=\bigcap_{i=1}^{N} \uparrow\left[c_{i}, d_{i}\right] \chi_{\left(u_{i}, v_{i}\right)} .
$$

Proposition 5.5. Suppose that, for $n \in \mathbb{N}$,

$$
v_{n}:=\sum_{i=1}^{K_{n}} r_{i}^{n} \delta_{a_{i}^{n}},
$$

as constructed in Sec. 4. Moreover, suppose that, for each $n \in \mathbb{N}$, $i=1, \ldots, K_{n}, e(\mu)\left(\uparrow a_{i}^{n}\right)$ is a computable number. Then for each basic open set $V, v_{n}(\square V)$ is computable.

Proof. The valuation $v_{n}(\square V)$ is given by

$$
v_{n}(\square V)=\sum_{\uparrow a_{i}^{n} \subseteq \square V} r_{i}^{n} .
$$

As we are using a basis of rational (or dyadic) intervals, it is clear from the characterisation of the way-below relation given in Proposition 2.1 that the predicate $\uparrow a_{i}^{n} \subseteq \square V$ is decidable. By Theorem 5.4, each $r_{i}^{n}$ is a computable number. The sum of at most $K_{n}$ computable numbers $r_{i}^{n}$ is computable. The result follows.

\section{Brownian motion and Wiener measure}

We shall now present an application to one of the most important processes in stochastic analysis, the Brownian motion, and the associated measure, the Wiener measure. This measure, introduced by $\mathrm{N}$. Wiener in [37], was the first major extension of integration theory beyond a finite-dimensional setting. We also present an alternative, domain-theoretic, view of the computability of this measure to that of [13].

We would like to approximate the Wiener measure of the compactopen set $R:=V([u, v] ;(a, b))$. We begin by considering the case when $u=0, a<0<b$, and employ the following theorem:

Theorem 6.1. [20, Theorem 3.23] Let $W_{t}$ be the Brownian motion on $T=[0,1], t \in T, m_{t}:=\min _{0 \leq s \leq t} W_{t}, M_{t}:=\max _{0 \leq s \leq t} W_{t}$. The joint distribution of the processes $W_{t}, m_{t}, M_{t}$ is given by

$$
\mathbb{P}\left[a<m_{t} \leq M_{t}<b \text { and } W_{t} \in A\right]=\int_{A} k(y) d y,
$$

where $A \subseteq \mathbb{R}$ is a measurable set,

$$
k(y):=\sum_{n=-\infty}^{\infty} p_{t}(2 n(b-a), y)-p_{t}(2 a, 2 n(b-a)+y),
$$

and

$$
p_{t}(x, y):=\frac{1}{\sqrt{2 \pi t}} e^{-(y-x)^{2} /(2 t)}
$$

A proof of this result appears in [15, pp. 26-27]. The equation (8) is due to Paul Lévy [26]. The result is extended and investigated numerically in [22] with applications in mathematical finance. For our purposes it suffices to consider the case when $A=(a, b)$. It is convenient to regard (8) as a special case of the following function of two variables,

$$
\begin{gathered}
x \in(a, b), \\
y \in(a-x, b-x) \subseteq(a-b, b-a): \\
k(x, y):=\sum_{n=-\infty}^{\infty} p_{t}(2 n(b-a), y)-p_{t}(2(a-x), 2 n(b-a)+y) .
\end{gathered}
$$

In (8), $x$ is 0 . By introducing $x$ we are effectively allowing the Brownian motion an intercept from the origin. This will be utilised in the sequel. To make the dependence on $a, b$, and $t$ explicit, we shall also write $k(x, y ; a, b ; t)$.

Proposition 6.2. The series (9) converges uniformly (as a series of functions of $x$ and $y$ ) and absolutely (as a series of numbers, for all $x$ and $y$ ). As a special case, (8) converges uniformly (as a series of functions of $x$ ) and absolutely (as a series of numbers, for all $x$ ).

Proof. Fix $\epsilon>0$. Let us consider the $M$-tails

$$
\begin{aligned}
k_{1}(y ; M) & :=\sum_{n \geq M} p_{t}(2 n(b-a), y), \\
k_{2}(y ; M) & :=\sum_{n \leq-M} p_{t}(2 n(b-a), y), \\
k_{3}(x, y ; M) & :=\sum_{n \geq M} p_{t}(2(a-x), 2 n(b-a)+y), \\
k_{4}(x, y ; M) & :=\sum_{n \leq-M} p_{t}(2(a-x), 2 n(b-a)+y) .
\end{aligned}
$$

By Chebyshev's inequality, for any random variable $X$ with finite mean $y$ and finite variance $t$,

$$
\mathbb{P}[|X-y| \geq \alpha t] \leq \frac{1}{\alpha^{2}}
$$


for any $\alpha \in \mathbb{R}^{+}$. Choose $\alpha=\frac{1}{\sqrt{\epsilon}}$. Then the inequality becomes

$$
\mathbb{P}\left[|X-y| \geq \frac{t}{\sqrt{\epsilon}}\right] \leq \epsilon .
$$

Set

$$
N_{1}(y):=\left\lceil\frac{(t / \sqrt{\epsilon})+y}{2(b-a)}\right\rceil .
$$

By this inequality and the symmetry of the normal distribution around its mean,

$$
\int_{N_{1}(y)}^{\infty} p_{t}(2 j(b-a), y) d j \leq \frac{\epsilon}{2}<\epsilon .
$$

For $n \geq N_{1}(y)$, the series of integrable positive-valued functions

$$
\sum_{j \geq n} p_{t}(2 j(b-a), y)
$$

is monotonically decreasing and bounded above by this integral, therefore the limit of the series exists and

$$
\sum_{j \geq n} p_{t}(2 j(b-a), y) \leq \int_{N_{1}(y)}^{\infty} p_{t}(2 j(b-a), y) d j<\epsilon .
$$

By the same reasoning, for all $n \leq-N_{2}(y)$,

$$
\begin{gathered}
N_{2}(y):=\left\lceil\frac{(t / \sqrt{\epsilon})-y}{2(b-a)}\right\rceil, \\
\sum_{j \leq n} p_{t}(2 j(b-a), y) \leq \int_{-\infty}^{N_{2}(y)} p_{t}(2 j(b-a), y) d j<\epsilon .
\end{gathered}
$$

We apply Chebyshev's inequality again, this time to a random variable $Y$ with finite mean $2 n(b-a)+y$. For any $\beta \in \mathbb{R}^{+}$,

$$
\mathbb{P}[|Y-2 n(b-a)-y| \geq \beta t] \leq \frac{1}{\beta^{2}} .
$$

Choose $\beta=\frac{1}{\epsilon}$. Then the inequality becomes

Set

$$
\mathbb{P}\left[|Y-2 n(b-a)-y| \geq \frac{t}{\sqrt{\epsilon}}\right] \leq \epsilon .
$$

$$
N_{3}(x, y):=\left\lceil\frac{(t / \sqrt{\epsilon})-y+2(a-x)}{2(b-a)}\right\rceil .
$$

By this inequality and the symmetry of the normal distribution around its mean,

$$
\int_{N_{3}(x, y)}^{\infty} p_{t}(2(a-x), 2 n(b-a)+y) d j \leq \frac{\epsilon}{2}<\epsilon .
$$

For $n \geq N_{3}(x, y)$, the series of integrable positive-valued functions

$$
\sum_{j \geq n} p_{t}(2(a-x), 2 n(b-a)+y)
$$

is monotonically decreasing and bounded above by this integral, therefore the limit of the series exists and

$$
\begin{gathered}
\sum_{j \geq n} p_{t}(2(a-x), 2 n(b-a)+y) \leq \\
\int_{N_{3}(x, y)}^{\infty} p_{t}(2(a-x), 2 n(b-a)+y) d j<\epsilon .
\end{gathered}
$$

By the same reasoning, for all $n \leq-N_{4}(x, y)$,

$$
N_{4}(x, y):=\left\lceil\frac{(t / \sqrt{\epsilon})+y-2(a-x)}{2(b-a)}\right\rceil,
$$

$$
\begin{gathered}
\sum_{\substack{j \leq n \\
N_{4}(x, y)}} p_{t}(2(a-x), 2 n(b-a)+y) \leq \\
\int_{-\infty}^{N_{t}} p_{t}(2(a-x), 2 n(b-a)+y) d j<\epsilon .
\end{gathered}
$$

Note that, for all $x$ and $y$,

$$
N_{1}(y), N_{2}(y), N_{3}(x, y), N_{4}(x, y) \leq N:=\left\lceil\frac{(t / \sqrt{\epsilon})+3(b-a)}{2(b-a)}\right\rceil,
$$

thus

$$
k_{1}(y ; N), k_{2}(y ; N), k_{3}(x, y ; N), k_{4}(x, y ; N)<\epsilon .
$$

We have shown that each of the series

$$
k_{1}(y ; 0), k_{2}(y ; 1), k_{3}(x, y ; 0), k_{4}(x, y ; 1)
$$

converges uniformly as functions of $x$ and $y$ and, since these are series of positive terms, they also converge absolutely. As a consequence of absolute convergence we can rearrange the terms in summation, and

$$
k(x, y)=k_{1}(y ; 0)+k_{2}(y ; 1)-k_{3}(x, y ; 0)-k_{4}(x, y ; 1)
$$

likewise converges uniformly and absolutely.

As a consequence of uniform convergence, (8) can be integrated term by term. Hence the following result, which is used in [22] and elsewhere in the literature on mathematical finance:

Proposition 6.3. The Wiener measure of the compact-open set $V([0, v] ;(a, b))$ with $a<0<b, \mu_{W}(a, b ; v)$, is given by

$$
\sum_{n=-\infty}^{\infty}\left\{\Phi\left(\frac{a-(2 n+1) \delta}{\sqrt{v}}\right)+\Phi\left(\frac{b-2 n \delta}{\sqrt{v}}\right)-2 \Phi\left(\frac{a-2 n \delta}{\sqrt{v}}\right)\right\}
$$

where $\delta=b-a$ and $\Phi$ is the cumulative distribution function of the standard normal distribution.

Proof.

$$
\begin{aligned}
\mu_{W}(a, b ; v) & =\mathbb{P}\left[a<m_{v} \leq M_{v}<b\right] \\
& =\mathbb{P}\left[a<m_{v} \leq M_{v}<b \text { and } W_{v} \in[a, b]\right] \\
& =\int_{[a, b]} k(y) d y \\
& =\int_{a}^{b}\left\{\sum_{n=-\infty}^{\infty} p_{v}(2 n \delta, y)-p_{v}(2 a, 2 n \delta+y)\right\} d y .
\end{aligned}
$$

Now, $p_{t}(q, r)=\frac{1}{\sqrt{t}} \phi\left(\frac{r-q}{\sqrt{t}}\right)$, where $\phi$ is the probability density function of the standard normal random variable, and

$$
\int_{a}^{b} p_{t}(q, r) d r=\Phi\left(\frac{b-q}{\sqrt{t}}\right)-\Phi\left(\frac{a-q}{\sqrt{t}}\right)
$$


where $\Phi$ is the cumulative density function of the standard normal random variable, and so

$$
\begin{aligned}
& \mu_{W}(a, b ; v) \\
& \stackrel{\text { uniform convergence }}{=} \sum_{n=-\infty}^{\infty}\left\{\int_{a}^{b} p_{v}(2 n \delta, y)-p_{v}(2 a, 2 n \delta+y) d y\right\} \\
& =\sum_{n=-\infty}^{\infty}\left\{\int_{a}^{b} p_{v}(2 n \delta, y) d y-\int_{a}^{b} p_{v}(2 a, 2 n \delta+y) d y\right\} \\
& =\sum_{n=-\infty}^{\infty}\left\{\left[\Phi\left(\frac{b-2 n \delta}{\sqrt{v}}\right)-\Phi\left(\frac{a-2 n \delta}{\sqrt{v}}\right)\right]-\right. \\
& =\sum_{n=-\infty}^{\infty}\left\{\Phi\left(\frac{a-(2 n+1) \delta}{\sqrt{v}}\right)+\Phi\left(\frac{b-2 n \delta}{\sqrt{v}}\right)-2 \Phi\left(\frac{a-2 n \delta}{\sqrt{v}}\right)\right\} .
\end{aligned}
$$

In Proposition 6.3 we have given the formula for the measure of a special case of a subbasic open set. We shall generalise this (in equation 10) to a general basic open set.

\section{Proposition 6.4. The partial function}

$$
k:(a, b) \times(a-b, b-a) \rightarrow \mathbb{R}
$$

with $k(x, y)$ given by (9) for

$$
y \in(a-x, b-x) \subseteq(a-b, b-a)
$$

and undefined otherwise is computable for all $x \in(a, b)$ and $y \in(a-x, b-x)$ with $a, b$ rational or dyadic.

Proof. For each positive integer $M$, define the $M$-tail, $k(x, y ; M)$, of $k(x, y)$, by

$$
\sum_{n=M,-M, M+1,-M-1, \ldots} p_{t}(2 n \delta, y)-p_{t}(2(a-x), 2 n \delta+y),
$$

where $\delta=b-a$. The other $M$-tails are as defined in Proposition 6.2. Following the proof of that proposition, for any positive integer $m$, set $\epsilon^{\prime}=1 / 2^{m}$ and $\epsilon=\epsilon^{\prime} / 4$. Set

$$
N_{m}>\left\lceil\frac{(t / \sqrt{\epsilon})+3(b-a)}{2(b-a)}\right\rceil .
$$

Then

$$
k_{1}\left(y ; N_{m}\right), k_{2}\left(y ; N_{m}\right), k_{3}\left(x, y ; N_{m}\right), k_{4}\left(x, y ; N_{m}\right)<\epsilon .
$$

Then

$$
\begin{aligned}
& \left|k\left(x, y ; N_{m}\right)\right| \\
& =\left|\left(k_{1}\left(y ; N_{m}\right)+k_{2}\left(y ; N_{m}\right)\right)+\left(-k_{3}\left(x, y ; N_{m}\right)-k_{4}\left(x, y ; N_{m}\right)\right)\right| \\
& \leq k_{1}\left(y ; N_{m}\right)+k_{2}\left(y ; N_{m}\right)+k_{3}\left(x, y ; N_{m}\right)+k_{4}\left(x, y ; N_{m}\right) \\
& <4 \epsilon=\epsilon^{\prime}=1 / 2^{m} .
\end{aligned}
$$

That is,

$$
-\frac{1}{2^{m}}<k\left(x, y ; N_{m}\right)<\frac{1}{2^{m}} .
$$

Notice that each term in $k\left(x, y ; N_{m}\right)$ is computable (see, for example, [28]). The result follows by the second part of [36, Theorem 4.3.8].

Let $V:=V\left(K_{1}, \ldots, K_{n} ; U_{1}, \ldots, U_{n}\right), n \in \mathbb{N}^{+}$be a basic open set (Figure 2). In our context, where $X$ will be a nonempty compact

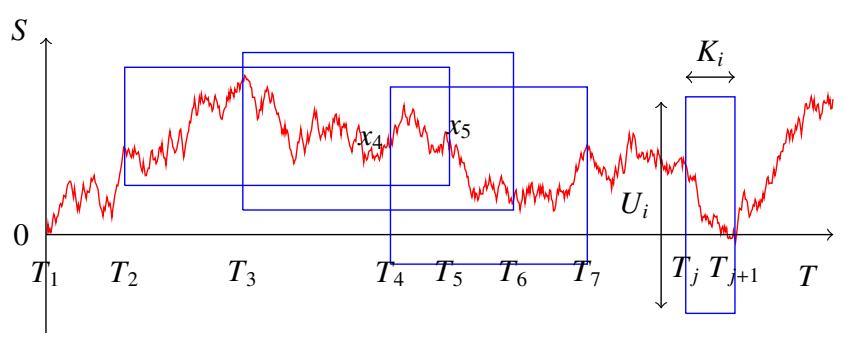

Figure 2. Wiener measure of a basic set

interval, $X \subseteq \mathbb{R}$, the basic open set $V \subseteq C(X, Y)$ induces a partition of $X$ :

$$
\mathcal{T}(V):=\{\min X, \max X\} \cup \bigcup_{i=1}^{n}\left\{\min K_{i}, \max K_{i}\right\} .
$$

We shall regard it as a naturally ordered (in ascending order) tuple containing $|\mathcal{T}(V)| \leq 2(n+1)$ (distinct) elements and refer to its elements as $T_{1}, \ldots, T_{|\mathcal{T}|}$, where the dependence on $V$ is implicit.

For $i=1, \ldots,|\mathcal{T}|-1$, define

$$
f_{i}(x, y):= \begin{cases}k\left(x, y ; L_{i}, R_{i} ; \Delta t_{i}\right) & \text { if }\left[T_{i}, T_{i+1}\right] \subseteq \bigcup_{j=1}^{n} K_{j}, \\ \frac{1}{\sqrt{\Delta t_{i}}} \phi\left(\frac{y-x}{\sqrt{\Delta t_{i}}}\right) & \text { otherwise, }\end{cases}
$$

where $\phi$ is the standard normal density function, $\Delta t_{i}=T_{i+1}-T_{i}$,

$$
\left[L_{i}, R_{i}\right]:=\bigcap_{j=1}^{n}\left\{U_{j} \mid\left[T_{i}, T_{i+1}\right] \subseteq K_{j}\right\} .
$$

Then, using the properties of conditional probability,

$$
\begin{gathered}
\mu_{W}(V)=\int_{A_{1}} \int_{A_{2}} \ldots \int_{A_{|\mathcal{T}|-1}} f_{1}\left(x_{0}, x_{1}\right) f_{2}\left(x_{1}, x_{2}\right) \ldots \\
f_{|\mathcal{T}|-1}\left(x_{|\mathcal{T}|-2}, x_{|\mathcal{T}|-1}\right) d x_{1} d x_{2} \ldots d x_{|\mathcal{T}|-1},
\end{gathered}
$$

where $x_{0}=0$, for $i=1, \ldots,|\mathcal{T}|-1, A_{i}:=\bigcap_{j=1}^{n}\left\{U_{j} \mid T_{i+1} \in K_{j}\right\}-x_{i-1}$, utilising the convention that the intersection of an empty collection of sets is the universal set. Note that, when each of $K_{1}, \ldots, K_{n}$ is a singleton, the compact-open subset reduces to a point-open subset and the integral reduces to (1).

Recall that we have chosen the topological bases for $T=[0,1]$ and $S=\mathbb{R}$ so that they consist of either rational or dyadic open intervals. The following proof is an alternative to that constructed in [13]:

Proposition 6.5. The Wiener measure (10) of a basic open set is computable.

Proof. The product of computable functions is computable (the computability of the factors has been addressed in Proposition 6.4), therefore the integrand is computable. The computability of the integral follows from Corollary $6 \mathrm{c}$ in [32].

Corollary 6.6. Suppose that, for $n \in \mathbb{N}$,

$$
v_{n}:=\sum_{i=1}^{K_{n}} r_{i}^{n} \delta_{a_{i}^{n}},
$$

as constructed in Sec. 4 with $\mu$ fixed as the Wiener measure, $\mu_{W}$. Then $v_{0} \sqsubseteq v_{1} \sqsubseteq v_{2} \sqsubseteq \ldots$ is an effective chain and its supremum is in effect the Wiener measure, which is therefore a domaintheoretically computable stochastic process. 


\section{Conclusions and further work}

We have developed a domain-theoretic framework for Brownian motion and general continuous stochastic processes. This creates plenty of scope for further work. For example, R-integration [5], with its extension to more general topological spaces as in [17] and [25], and domain-theoretic Lebesgue-integration [10] can be applied naturally to our construction, thus giving rise to a new alternative to Monte-Carlo simulation for computing probabilistic expectations. Sec. 4 motivates an investigation into the computational aspects of the procedure for approximating stochastic processes as partial stochastic processes. For example, given $\mu$, what is the optimal choice of basis (equation (5)) and how should it be ordered? It is apparent that these choices depend on the rate of growth of the stochastic process. In the case of the Brownian motion, the almost sure asymptotic growth is given by the wellestablished upper-envelope results [29, Chapter 5].

\section{Acknowledgments}

We would like to thank the anonymous reviewers and Tsz Lee for valuable corrections and suggestions.

\section{References}

[1] Samson Abramsky and Achim Jung. Domain theory. In Handbook of logic in computer science (vol. 3), pages 641-761. Oxford University Press, Inc., 1994.

[2] Mauricio Alvarez-Manilla, Abbas Edalat, and Nasser SahebDjahromi. An extension result for continuous valuations. Journal of the London Mathematical Society, 61(2):629-640, April 2000.

[3] Alexandre Joel Chorin. Accurate evaluation of Wiener integrals. Mathematics of Computation, 27(121):1-15, 1973.

[4] George Davie and Willem L. Fouché. On the computability of a construction of Brownian motion. Mathematical Structures in Computer Science, 23(6):1257-1265, December 2013.

[5] Abbas Edalat. Domain theory and integration. Theoretical Computer Science, 151(1):163-193, November 1995.

[6] Abbas Edalat. Domain theory in stochastic processes. In Logic in Computer Science (LICS), 1995.

[7] Abbas Edalat. Dynamical systems, measures, and fractals via domain theory. Information and Computation, 120(1):32-48, July 1995.

[8] Abbas Edalat. Domains for computation in mathematics, physics and exact real arithmetic. Bulletin of Symbolic Logic, 3(4):401-452, 1997.

[9] Abbas Edalat. When Scott is weak on the top. Mathematical Structures in Computer Science, 7(5):401-417, October 1997.

[10] Abbas Edalat. A computable approach to measure and integration theory. Information and Computation, 207(5):642-659, May 2009.

[11] Abbas Edalat. A differential operator and weak topology for Lipschitz maps. Topology and its Applications, 157(9):1629-1650, June 2010.

[12] Thomas Erker, Martín Escardó, and Klaus Keimel. The way-below relation of function spaces over semantic domains. Topology and its Applications, 89(1-2):61-74, November 1998.

[13] Willem L. Fouché. Arithmetical representations of Brownian motion I. The Journal of Symbolic Logic, 65(1):421-442, March 2000.

[14] Willem L. Fouché. The descriptive complexity of Brownian motion. Advances in Mathematics, 155(2):317-343, November 2000.

[15] David Freedman. Brownian Motion and Diffusion. Springer-Verlag, 1983.

[16] Gerhard Gierz, K. H. Hofmann, K. Keimel, J. D. Lawson, M. Mislove, and D. S. Scott. Continuous Lattices and Domains. Number 93 in Encyclopedia of Mathematics and its Applications. Cambridge University Press, 2003.
[17] J. D. Howroyd. A domain-theoretic approach to integration in hausdorff spaces. London Mathematical Society Journal of Computation and Mathematics, 3:229-273, August 2000.

[18] Mathieu Hoyrup. Calculabilité, aléatoire et théorie ergodique sur les espaces métriques. $\mathrm{PhD}$ thesis, Ecole Doctorale de Sciences Mathématiques de Paris Centre, 2008.

[19] Claire Jones and Gordon D. Plotkin. A probabilistic powerdomain of evaluations. In Proceedings of the Fourth Annual Symposium on Logic in Computer Science (LICS), June 1989.

[20] Fima C. Klebaner. Introduction to Stochastic Calculus with Applications. Imperial College Press, 3 edition, 2012.

[21] I.M. Koval'chik. The Wiener integral. Russian Mathematical Surveys: Uspekhi Mat. Nauk, 18(1):97-134, 1963.

[22] Naoto Kunitomo and Masayuki Ikeda. Pricing options with curved boundaries. Mathematical Finance, 2(4):275-298, October 1992.

[23] Jimmie Lawson. Computation on metric spaces via domain theory. Topology and its Applications, 85:247-263, 1998.

[24] Jimmie D. Lawson. Spaces of maximal points. Mathematical Structures in Computer Science, 7(5):543-555, October 1997.

[25] Jimmie D. Lawson. Domains, integration and 'positive analysis' Mathematical Structures in Computer Science, 14(6):815-832, 2004.

[26] Paul Lévy. Processus stochastiques et mouvement brownien. Paris, Gauthier-Villars, 2 edition, 1965.

[27] Shoumei Li, Jungang Li, and Xiaohua Li. Stochastic integral with respect to set-valued square integrable martingales. Journal of Mathematical Analysis and Applications, 370:659-671, 2010.

[28] Takakazu Mori, Yoshiki Tsujii, and Mariko Yasugi. Computability of probability distributions and distribution functions. In Sixth International Conference on Computability and Complexity in Analysis (CCA 2009), 2009.

[29] Peter Mörters and Yuval Peres. Brownian Motion. Cambridge Series in Statistical and Probabilistic Mathematics. Cambridge University Press, 2010.

[30] Brent Øksendal. Stochastic Differential Equations: An Introduction with Applications. Universitext. Springer, 2000.

[31] V.I. Sobolev (originator). Wiener integral. Encyclopedia of Mathematics. URL: http://www. encyclopediaofmath.org/index.php? title=Wiener_integral\&oldid=17004.

[32] Marian Boykan Pour-El and Ian Richards. Computability and noncomputability in classical analysis. Transactions of the American Mathematical Society, 275:539-560, 1983.

[33] Dana Stewart Scott. Outline of a mathematical theory of computation. In Proceedings of the Fourth Annual Princeton Conference on Information Sciences and Systems, 1970.

[34] Barry Simon. Functional Integration and Quantum Physics. AMS Chelsea Publishing, 2 edition, 2005.

[35] Klaus Weihrauch. Computability on the probability measures on the borel sets of the unit interval. Lecture Notes in Computer Science: Automata Languages and Programming, 1256:166-176, 1997.

[36] Klaus Weihrauch. Computable Analysis: An Introduction. Texts in Theoretical Computer Science, An EATCS Series. Springer-Verlag Berlin Heidelberg, 2000.

[37] Norbert Wiener. Differential space. Journal of Mathematical Physics, 2:131-174, 1923.

[38] Yongcheng Wu and Decheng Ding. Computability of measurable sets via effective metrics. Mathematical Logic Quarterly, 51(6):543-559, November 2005.

[39] Yongcheng Wu and Decheng Ding. Computability of measurable sets via effective topologies. Archive for Mathematical Logic, 45(3):365379, March 2006.

[40] Jinping Zhang. Interval-valued stochastic processes and stochastic integrals. In Second International Conference on Innovative Computing, Information and Control (ICICIC), page 466, 2007. 